\title{
Perceived challenges for adopting the Personal Health Record (PHR) at Ministry of National Guard Health Affairs (MNGHA) -
} Riyadh

\author{
Al-Sahan A MSc (HI) ${ }^{1}$, Saddik B PhD, $\mathrm{MPH}^{1}$. \\ 1. College of Public Health and Health Informatics, King Saud Bin Abdulaziz University for Health \\ Sciences (KSAU-HS)
}

\begin{abstract}
Background: The Personal Health Record (PHR) is an electronic record that allows patients to maintain, manage and access their health information in one secure location. However, despite these potential capabilities, the adoption rate of the PHR has been slow due to various challenges.

Objectives: This study, being the first of its kind in Saudi Arabia, investigates the perceived barriers and /or challenges for PHR adoption in the Ministry of National Guard Health Affairs (MNGHA). The study explored perceived barriers and /or challenges from two different perspectives; a technical perspective and a social perspective.
\end{abstract}

Methods: The study was conducted using a mixed methods approach. A cross-sectional study design using a questionnaire was used to measure patients' perceptions of the PHR and a qualitative approach through focus groups was used to capture comments and opinions from technical personnel for perceived technical barriers to PHR adoption.

Result: Results from 424 patients revealed a positive perception for PHR adoption with almost all of the participants $(96.7 \%)$ indicating interest in using the PHR and the majority $(\mathbf{7 3 . 3 \% )}$ expressing no confidentiality concerns for the online accessibility of their health information. Patients with higher levels of education indicated higher interest in using the PHR and expressed more concern with confidentiality than patients with lower levels of education. However, the majority of patients (78.3\%) expressed their lack of awareness of existing patient e-services on the MNGHA website. The themes that emerged from the focus groups reinforced lack of awareness of e-services as a potential barrier for PHR adoption as well as the role of policy in the regulation and business process for PHR adoption.

Conclusion: This study has highlighted the perceived challenges and barriers for adoption of the PHR in MNGHA-Riyadh. In order to ensure an efficient PHR with a strong adoption rate, effective steps need to be undertaken by building PHR awareness as well as setting clear guidelines and regulations from policy makers.

Keywords: PHR adoption, Challenges affecting PHR adaption, PHR challenges, PHR barriers, Personal Health Record, PHR

Correspondence: basema.saddik@gmail.com, aljohara_jes@hotmail.com

DOI: $10.5210 /$ ojphi.v8i3.6845 
Copyright @2016 the author(s)

This is an Open Access article. Authors own copyright of their articles appearing in the Online Journal of Public Health Informatics. Readers may copy articles without permission of the copyright owner(s), as long as the author and OJPHI are acknowledged in the copy and the copy is used for educational, not-for-profit purposes.

\section{Introduction}

The Personal Health Record (PHR) has been used by patients as a tool for continuity of healthcare for decades. In the past, the PHR was viewed as a paper-based form retained by patients in the form of laboratory results, prescription notes and other forms of health related documents [1-5]. Nowadays with the rapid growth of technology within the healthcare sector, the old concept of the PHR has been replaced with an Electronic PHR. According to Markle Foundation, the PHR has been defined as, "an electronic application through which individuals can access, manage and share their health information, and that of others for whom they are authorized, in a private, secure, and confidential environment" [6-9]. There are different types of PHRs: the Standalone or the Thethered. The Standalone PHR is through commercial online websites that allow patients to record their health behaviors. On the other hand, the Tethered PHR is usually linked to a specific organization through its Electronic Medical Record (EMR) to ensure patient's access (as read only) to their health information [2-6, 10-12]. In theory, the PHR has the capabilities that allow patients to keep track of their health information, monitor and manage their illness especially in cases of chronic diseases, enhance efficiencies in appointment scheduling, medication refills, and improve the communication with healthcare providers for better quality of care [1-6, 9-13]. However, despite the reported potential capabilities of the PHR, the adoption rate has been low [4,8-14] due to a number of reported challenges and barriers such as interoperability, policy, confidentiality, privacy concerns, security issues, lack of awareness of services, inadequate internet access, computer literacy, computer anxiety and health literacy [3, 5, 7-10, 12-18]. Policy also plays an important role in the establishment of the PHR in terms of business processes, regulations and standards which have also been reported to impact on the adoption rates of the PHR $[8,9]$. In the United States three case studies identified policy barriers in terms of what information the PHR should include and under which circumstances the information within the PHR should be released and shared [15]. Halamka et.al, reports that based on state laws certain restrictions were exercised for releasing certain results to the patients such as in the case of Human Immunodeficiency Virus (HIV) [15]. Interoperability has also been reported as a barrier to the effective adoption of the PHR for the exchange of health information among multiple healthcare systems [3, 5, 7-9, 13, 15]. Moreover, the electronic exchange of health information indicates major concerns regarding the issue of protecting patients' confidentiality, privacy and PHR security during the online transmission of health information $[3,5,8,9,12-15,17,18]$. According to a US national survey around two-thirds of the participants had concerns about the privacy and the security of their health information [3, 17]. Another barrier to adoption of the PHR which has been reported in the literature is the lack of awareness of the PHR. A study in South Africa reported that almost all of the citizens were unaware of the existence of the PHR or what it could be used for [13]. Educating citizens on the benefits of the PHR and promoting it as an effective tool for healthcare, has been found to reduce resistance to change [14] as well as address concerns associated with using computers. Elderly and computer 
illiterate patients are less keen on adopting the PHR due to computer anxiety and health literacy concerns $[13,16]$ and healthcare providers should be able to address these concerns early on in order to effectively manage and enhance the adoption of the PHR [16]. Although the challenges and barriers to PHR adoption have been highlighted in the literature, it is still not clear what these challenges are within the Saudi society. The Ministry of National Guard Health Affairs (MNGHA) in Saudi Arabia is in the implementation phase of the PHR with the collaboration of the National e-government initiative. Although the PHR is expected to enhance patient care, its full implementation is also expected to face various challenges as per previously reported experiences. This study aims to investigate the perceived barriers for PHR adoption which may also be challenges for implementing an efficient PHR in MNGHA, as well as measure the extent of patients' interests towards PHR adoption according to their health status, and identify patients' needs in terms of data and services needed to be available in the PHR.

\section{Methodology}

\section{Study Design}

In order to ensure comprehensiveness, this study used a mixed methods approach to investigate the perceived barriers and challenges to PHR adoption. A descriptive quantitative approach was used to measure how patients perceived the PHR from a social perspective and a qualitative approach was used to capture comments and opinions from technical personnel for perceived technical barriers to PHR adoption.

\section{Study Setting}

The study was conducted at the Ministry of National Guard Health Affairs (MNGHA) King AbdulAziz Medical City (KAMC). MNGHA consists of different medical cities comprising of four hospitals and sixty primary and secondary health centers scattered in five regions around the kingdom of Saudi Arabia (Riyadh, Jeddah, Medina, Dammam and Al-Ahsa)[19]. The primary and the largest medical city is King Abdul-Aziz Medical City (KAMC) located in Riyadh. This study was conducted at the Ambulatory Care Center (ACC) of KAMC at King Fahd hospital. The ACC is one of the central centers in the hospital including pharmacy, employee clinic, biobank laboratory and other specialized clinics such as Pediatric, Neurology, Oncology, Hematology and other clinics.

\section{Selection and Description of Participants}

In order to clearly understand the perceived challenges for PHR adoption from different perspectives, two groups were selected to participate in the study; a patient group and a technical group. Convenience sampling was used for both groups who met the inclusion criteria. For the patient group the inclusion criteria included all patients who attended the MNGHA ACC between the ages of 20-60. This age group was selected because individuals less than 20 are usually under the guardianship of the head of the family whose contact details are used for registration and hence do not have direct access to their medical records. Furthermore, it is believed that patients over the age of 60 also have their primary carers' contact numbers registered and their records would usually be accessed by the carer and not the patient themselves. This age group was also selected based on the criteria highlighted in the study 
conducted by (Hamlaka et al.,) [15] which defined record access into three categories; 1) full access to the parents for those patients who are less than 12 years old. 2) Balanced access between the patient from age 12 to 18 and his/her parents but some of the information may be restricted to one of them. 3) Full access and control of the record for those who are 18 years old and older [8]. For the technical group, the inclusion criteria for technical staff included staff who were working in the Information Systems \& Informatics Divisions (ISID) and were involved in the development and implementation of the PHR project at MNGHA comprising of managers and a representative from the national e-government initiative who came from different regions within MNGHA ; Riyadh, Al-hasa and Jeddah.

\section{Ethical Considerations}

The research study protocol was approved by King Abdullah International Research Center (KAIMRC) through the International Review Board (IRB) protocol number (SP14/049). All participants in the research study were provided with information prior to participation and informed consent was obtained. No participant was identified and confidentiality was ensured for all participants. All data were reported in aggregate form and no participant was identified in any way.

\section{Data Collection}

There were two data collection methods used in this study, a questionnaire and two focus groups. The questionnaire was developed to answer the research question and address the objectives of the study. It comprised of 19 questions in total which covered demographic information, health status, concerns about data confidentiality, computer literacy or internet access and seven specific questions on patients' perceptions of the PHR which were borrowed from another validated survey [20]. The questionnaire was translated to Arabic and back translated to English to ensure reliability of translation. Furthermore, to test the validity and reliability of the questions and to ensure that the objectives of the study were met, the questionnaire was piloted among 31 participants who were patients in MNGHA and were eligible to participate according to the above mentioned inclusion criteria. Based on the pilot testing, the questionnaire was modified to improve clarity. Data collection began in mid-September 2014 until the end of October by visiting the ACC three days a week in the afternoon excluding the national day and hajj holiday. Data were collected in person by inviting patients in the waiting area at the employees' clinic and other clinics within the ACC. The questionnaire along with the consent form were distributed with a brief explanation about the purpose of the questionnaire and clarification on confidentiality concerns. However, in the case of elderly or illiterate patients who could not complete the survey themselves, the data were collected either through their carers or by asking them directly in a secluded area to ensure their confidentiality.

As for the focus groups, these were conducted to illicit participants' opinions on the perceived challenges for implementing the PHR from a technical perspective including technical issues, policies, guidelines and/or other regulations. Two focus groups were conducted by the researcher in the presence of a moderator and lasted for one hour per group; the first focus group included four participants and was held in the middle of November 2014 in an office at the convention center in King Saud Bin Abdul-Aziz University for Health Sciences in Riyadh. The second focus group contained three participants and was held beginning of December 2014 at one of the 
participant's office in Riyadh. All participants provided their informed consent and permission for an audio recording of the session. The discussions within both focus groups were guided by a list of open-ended questions which addressed the specific themes of the research objectives. In addition to the focus groups an individual in-depth interview was conducted by the researcher with a member of the technical expert team to confirm a certain point raised in the focus group about integration.

\section{Sample Size}

The sample size for the patient group was calculated based on the average number of patients who visited the ACC in 2013 which was approximately 70644 per month. Therefore assuming P $<0.05$ as maximum variability with $95 \%$ confidence interval and $5 \%$ precision, the sample size required was calculated at 385 In total 454 patients were included in the study, however, 30 patient responses were excluded due to incomplete responses, inconsistent answers or were not eligible based on the inclusion criteria set for the study. As for the focus groups with technical staff, it was expected that 6-10 participants would be included in each focus group. However due to logistical issues, time constraints and the inability to agree on mutually convenient times for all the invited focus group members, only 7 members of the technical team were able to participate in the focus groups and were divided into two focus group sessions.

\section{Data Analysis}

Data from the 19 questions were coded, entered and analyzed using SPSS software v.20.0. Descriptive data were analyzed using frequencies and percentages and Chi-square analysis was used to measure associations and correlations between variables of interest. Level of significance was determined by a $P$ value equal to or less than $0.05(\mathrm{p} \leq 0.05)$. As for the qualitative data collected from the focus groups, each focus group session was recorded and transcribed verbatim and thematic analysis was done by identifying, coding and reporting recurring themes within the data according to the categories that were highlighted as being specific challenges or barriers for PHR adoption.

\section{Results}

\section{Questionnaire Findings}

\section{Demographic Information and Perceived Health Status}

The sample mostly comprised of females and those of Arabic origin. The majority perceived themselves to be healthy, where only few reported suffering from a chronic disease. Very few reported attaining a low educational level (illiterate, literate and completing elementary school). The age distribution among the study sample was somewhat similar, whereby individuals were predominantly between 20 and 39 years of age (Table 1). 
Table 1 - Demographic and perceived health status on study participants $(n=424)$

\begin{tabular}{|c|c|}
\hline \multicolumn{2}{|c|}{ Demographic and Perceived Health Status N (\%) } \\
\hline \multicolumn{2}{|l|}{ Nationality } \\
\hline Arabian & $366(86.3 \%)$ \\
\hline Non-Arabian & $58(13.7 \%)$ \\
\hline \multicolumn{2}{|l|}{ Gender } \\
\hline Male & $135(31.8 \%)$ \\
\hline Female & $289(68.2 \%)$ \\
\hline \multicolumn{2}{|l|}{ Age (years) } \\
\hline $20-29$ & $140(33.0 \%)$ \\
\hline $30-39$ & $132(31.1 \%)$ \\
\hline $40-49$ & $81(19.1 \%)$ \\
\hline $50-60$ & $71(16.7 \%)$ \\
\hline \multicolumn{2}{|l|}{ Educational Level } \\
\hline Illiterate & $22(5.2 \%)$ \\
\hline Literate & $11(2.6 \%)$ \\
\hline Elementary & $9(2.1 \%)$ \\
\hline Intermediate & $31(7.3 \%)$ \\
\hline High School & $81(19.1 \%)$ \\
\hline Diploma & $4(0.9 \%)$ \\
\hline University & $205(48.3 \%)$ \\
\hline Higher Education & $61(14.4 \%)$ \\
\hline \multicolumn{2}{|l|}{ Perceived Heath Status } \\
\hline Healthy & $289(68.2 \%)$ \\
\hline Not healthy with chronic disease & $84(19.8 \%)$ \\
\hline Not healthy with other medical condition & $51(12.0 \%)$ \\
\hline
\end{tabular}

\section{Technology Use}

The majority of participants reported using smart devices, using computers and browsing the internet, however only 16\% accessed e-services from the MNGHA website (Table 2). The leading and most common reason reported from patients who reported using the internet but not accessing patients' e-services from the MNGHA website was their lack of awareness of the 
services provided (78.3\%). Moreover, (20.1\%) reported not knowing how to access these services and only (11.0\%) claimed not to be interested.

Table 2- Technology use among study participants $(n=424)$

\begin{tabular}{|l|l|}
\hline Technology Use & N (\%) \\
\hline Use of Internet $*$ & $377(95.9 \%)$ \\
\hline Yes & $16(4.1 \%)$ \\
\hline No & \\
\hline Use of Smart devices & $391(92.2)$ \\
\hline Yes & \\
\hline Use of Computer & $342(80.7)$ \\
\hline Yes & $60(15.9 \%)$ \\
\hline Accessing patients' e-services at MNGHA website ** \\
\hline Yes & $317(84.1 \%)$ \\
\hline No &
\end{tabular}

* Use of internet was computed among those reported using computers or using smart devices. ** Accessing patients' services at MNGHA website was computed among those answering “yes” to use of internet.

\section{Perceptions about the PHR}

Few of the participants reported having copies of their health information (e.g., laboratory results, radiology results or others) and almost all of the participants reported an interest in having an online PHR. Confidentiality concerns were not perceived a threat for the majority of the participants (Table 3).

Only a minority (26.7\%) of participants reported they had confidentiality concerns about the online accessibility of their health information. Such concerns attributed to confidentiality and privacy (52.2\%) in addition to security concerns (21.2\%). Open ended responses for these questions retrieved responses such as "belief in patient privacy" or "my information should be accessible to me only and should be confidential" as well as, "it may be hacked" or "someone may play with the access”

Amongst the participants who reported interest in having an online PHR, the main information they felt necessary to be accessed were laboratory results, radiology results, medication lists or other data such as diagnosis, medical history, vital signs or medical reports $(91.7 \%, 82.9 \%$, $72.4 \%$ and $6.1 \%$ respectively). In addition, in terms of what services participants felt they would like to be available online, the main services reported were appointment scheduling (90.5\%), communication with the physician $(74.1 \%)$ while only $(0.5 \%)$ reported other services such as determining the physician or awareness about the disease diagnosed. (Results not presented in table) 
Table 3 - Perceptions about PHR on study participants $(n=424)$

\begin{tabular}{|l|l|}
\hline Participants' Perceptions about PHR & N (\%) \\
\hline Having copies of their health information \\
\hline Yes & $83(19.0 \%)$ \\
\hline No & $341(80.4 \%)$ \\
\hline Interest in online PHR & $257(60.6 \%)$ \\
\hline Very Interested & $107(25.2 \%)$ \\
\hline Interested & $46(10.8 \%)$ \\
\hline Somewhat Interested & $14(3.3 \%)$ \\
\hline Not interested & \\
\hline Confidentiality's Concerns & $113(26.7 \%)$ \\
\hline Yes & $311(73.3 \%)$ \\
\hline No &
\end{tabular}

Further analysis using a Chi-square test with $P$ value set at 0.05 was conducted to determine the significant differences between participants' demographics information (e.g., Age, gender, educational level,...etc.) and their perceptions on PHR confidentiality. A significant difference was found between confidentiality concern, participants' nationality, educational level, and their health status (Table 4). Patients who were of Arabic origin were more likely to have confidentiality concerns than patients who were non Arabs. Furthermore, patients who had higher education and those who perceived themselves as healthy had significantly higher concerns with confidentiality than patients who had lower levels of education and perceived themselves as not healthy. In terms of participant's interest in using the PHR, significant differences were found between PHR interest and educational level $p \leq 0.001$ (Table 5). Patients with higher education were significantly more interested in using the PHR than those with lower education levels.

Table 4- Participants' demographic information and perceived health status with their perceptions about PHR confidentiality

\begin{tabular}{|l|l|l|}
\hline & N (\%) & \multicolumn{2}{|l|}{ p-value } \\
\hline Concerns about confidentiality & $\mathbf{1 1 3}(\mathbf{2 6 . 7 )}$ & \multicolumn{2}{|l|}{} \\
\hline Nationality & \multicolumn{2}{|l|}{} \\
\hline Arabian & $76(67.2)$ & \\
\hline Non-Arabian & $37(32.7)$ & \multicolumn{2}{|l}{} \\
\hline Educational Level & & $\mathbf{0 . 0 0 3}$ \\
\hline Illiterate & $2(1.7)$ & \multicolumn{2}{|l|}{} \\
\hline
\end{tabular}




\begin{tabular}{|c|c|c|}
\hline Literate & $1(0.88)$ & \\
\hline Elementary & $1(0.88)$ & \\
\hline Intermediate & $3(2.6)$ & \\
\hline High School & $17(15)$ & \\
\hline Diploma & $1(0.88)$ & \\
\hline University & 62(54.8) & \\
\hline Higher Education & $26(23)$ & \\
\hline \multicolumn{3}{|l|}{ Perceived Health Status } \\
\hline Healthy & 90(79.6) & \multirow[t]{3}{*}{0.00} \\
\hline Not healthy with chronic disease & 15 (13.3) & \\
\hline Not health with other medical condition & $8(7)$ & \\
\hline
\end{tabular}

Table 5- Participants' educational level and PHR interest

\begin{tabular}{|c|c|c|c|c|c|}
\hline & \multicolumn{4}{|c|}{ Interest in PHR } & \multirow{3}{*}{$\begin{array}{l}\text { P- } \\
\text { value }\end{array}$} \\
\hline & \multicolumn{4}{|l|}{ N (\%) } & \\
\hline & $\begin{array}{l}\text { Very } \\
\text { interested }\end{array}$ & Interested & $\begin{array}{l}\text { Somewhat } \\
\text { interested }\end{array}$ & \begin{tabular}{|l} 
Not \\
interested
\end{tabular} & \\
\hline Total & $257(60.6)$ & $107(25.2$ & $46(10.8)$ & $14(3.3)$ & \\
\hline \multicolumn{6}{|l|}{ Educational level } \\
\hline Illiterate & $8(36.4)$ & $6(27.3)$ & $5(22.7)$ & $3(13.6)$ & \multirow[t]{8}{*}{0.001} \\
\hline Literate & $6(54.5)$ & $5(45.5)$ & $0(0.0)$ & $0(0.0)$ & \\
\hline Elementary & $3(33.3)$ & $3(33.3)$ & $0(0.0)$ & $3(33.3)$ & \\
\hline Intermediate & \begin{tabular}{|l}
$17(54.8)$ \\
\end{tabular} & 11(35.5) & $2(6.5)$ & $1(3.2)$ & \\
\hline High school & $46(56.8)$ & 20(24.7) & 12(14.8) & $3(3.7)$ & \\
\hline Diploma & $1(25.0)$ & $2(50.0)$ & $1(25.0)$ & $0(0.0)$ & \\
\hline University & 135(65.9) & $45(22.0)$ & $22(10.7)$ & $3(1.5)$ & \\
\hline Higher education & \begin{tabular}{|l|}
$41(67.2)$ \\
\end{tabular} & 15(24.6) & $4(6.6)$ & $1(1.6)$ & \\
\hline
\end{tabular}

\section{Focus Group Findings}

Discussion within the focus groups revolved around predefined questions used as a guide for discussion which continued until saturation was reached. All participants in both groups 
highlighted the importance of using the PHR as they considered it as a patient' rights. Additionally, when asked about the challenges in implementing PHR within MNGHA, the first group viewed the challenges to be related to lack of patients' awareness while participants in the other group did not view it as impossible indicating that "almost everyone is now using smart devices, even the elderly patients". Despite the challenges claimed, MNGHA is still in the initiation phase, it was highlighted that policy plays an important role when it comes to PHR content, particularly in terms of data and services. It is still not clear what type of data will be included in the PHR and what the regulations are for releasing particular results, as was mentioned "PHR content needs a task force that is well collaborated between policy makers". Moreover, it was reported that fully automated services depend on a clear workflow and on the currently used system which is acting as a limitation. When asked about the technical challenges (e.g., security, systems integration) all participants considered that the network within MNGHA to be secure. Moreover, participants added that if patients had access to their health information, the access will not be directly linked to the actual data center. In addition, there will be a multilayer authentication access similar to that used in bank transactions. Despite having a high secure authentication access, participants agreed that the actual process during registration does not guarantee confidentiality; one participant stated, "When we talk about the security, the technology is there but this also depends on the business process agreed by the decision makers which can be considered as a challenge".

Regarding integration, participants agreed that there are no major challenges within MNGHA. However, participants felt that format of data played a role in integration. One participant stated, "it depends on the data availability in the systems, whether it is in a paper record or not structured (e.g., physician report) where it acts as a challenge to extract all needed data and change their format". Finally, when asked about the potential challenges that may emerge after the implementation of the PHR the participants within the second focus group indicated "the resistance to change from the end users whether healthcare providers or patients could hinder the adoption of the PHR, for example any test result should be first approved by the physician before being submitted in the PHR. Such a process may be delayed, so this may be considered a reason for healthcare providers' resistance ..... that resistance usually has been faced regardless of the type of the system”.

\section{Discussion}

This study, being the first of its kind in Saudi Arabia, highlights the perceived barriers and /or challenges for PHR adoption in MNGHA. From a social perspective, this study was not different to other studies and found that almost all participants browsed the internet whether they used computers or smart devices. This indicates a potential strength for PHR adoption in MNGHA among frequent users of the internet and has been previously reported as a positive indicator for potential PHR adoption [12].

As for the usage of smart devices, our study also found that the majority of participants reported regular use of smart devices which has also been reported as a positive indicator for participants' willingness towards using mobile PHR (mPHR) [14]. However, despite the use of internet and smart devices in our study population, the majority of participants did not use MNGHA patient e-services with the majority reporting that they were not aware such services existed. This lack of awareness impacts negatively on PHR adoption and is potentially a significant barrier to 
adoption as highlighted in previous studies [14] and should be an area of concern and action once implementation of PHR is complete at MNGHA. Furthermore, the majority of patients reported not having copies of their health information also indicating difficulty or a lack of awareness for their ability to access their own health information.

Despite the lack of awareness about patients' e-services, what is surprising in our study findings is that almost all of the participants regardless of health status reported an interest in having a PHR which contradicts findings from other studies which found that patients with chronic conditions tend to be more interested in having a PHR than others [8, 14]. Even though our study population comprised mainly of young females, an interest in adopting a PHR is considered a strength for eventual PHR adoption especially with the difficulty of obtaining health information. However, our findings for PHR interest overall are consistent with other studies [5, 12, 17] regardless of such studies reporting that despite the major interest in the PHR the adoption rate remained slow.

The features of a PHR and the services accessed should be tailored to specific populations as these may differ among different patient and population groups. Patients in the Saudi context reported higher interest in services such as viewing test results and appointment scheduling in contrast to patients in the United States where appointment scheduling was not perceived as of much importance among patient groups [12, 17]. In the Saudi context, accessing test results and appointment scheduling online would alleviate some of the difficulties patients face with transportation in Saudi Arabia especially for females who represented the majority of our study population.

Regarding the participants' concerns about online accessibility and how this would impact on their privacy, confidentiality and security of their information, several studies in different countries like the United States [5, 8, 12, 13, 15, 17]; South Africa [14] and Canada [3,18], showed it to be a major challenge for PHR adoption. However, this was not the case in our study, where the majority of participants (73.3\%) showed no concerns. This could be explained by the culture of Saudi society particularly when it comes to dealing with health information and family involvement. Family is an important resource in patient care in Saudi Arabia and it may be very normal to share and view patient information with very limited concerns for privacy and confidentiality standards [21, 22].

From a technical perspective, our study showed that policy played an important role as a regulation or business process in the establishment of the PHR. Thus, it could be considered as a barrier that could slow the adoption of PHR. This finding is supported by other studies conducted in the United States $[8-10,13,15]$. Moreover, it was shown that integration at certain circumstances could present some challenges consistent with the literature $[3,5,7-9,13,15]$ in terms of integrating PHR with multiple healthcare systems indicating interoperability as a critical challenge as well as healthcare providers' readiness to adopt the PHR [3,5] which was also a concern highlighted by the focus group participants. Healthcare professionals' resistance to change was also perceived to hinder PHR adoption and it is encouraged that in order for the uptake of the PHR to be successful among patients, there needs to be enthusiasm from staff, healthcare professionals and patients [23]. Therefore we recommend that healthcare providers, policy makers, IT professionals and patients work together during the implementation of the PHR to ensure effective uptake and adoption of the PHR. 


\section{Limitations}

This study had several limitations including the cross-sectional design of the study and the sampling technique used. The study was conducted in MNGHA-Riyadh, and therefore inferences and generalizability to the whole population of MNGHA is limited. Given that data collection was done over a one month period, it did not capture a diverse population in terms of gender, age, educational level, health status, and technology usability. Almost all the participants were educated, healthy and were utilizing technology. The majority of our population were young females, however statistical data retrieved from MNGHA data centers [24] showed that the majority of patients who visited the ACC in 2014 were females and represented almost $60 \%$ of patients, therefore indicating that our study results are a true representation of the MNGHA ACC patients in 2014. Furthermore, focus group participants came from different regions within MNGHA and their views represent challenges all over the MNGHA. Focus group data were collected until saturation was achieved; hence ensuring good representation of participant's views. The self-reported health status is also considered a limitation of this study. Further research should assess health status of patients through their records rather than self-report to fully estimate the burden of disease in this population and assess the use of the PHR in chronic disease patients.

However, despite the limitations of this study, the results present new knowledge on the adoption of PHR in a different setting and the perceived challenges and barriers in Saudi Arabia which will help inform further in-depth studies in this area. Further research is required from the social perspective by including a diverse population in terms of their demographic information and other information from the different regions within MNGHA to attain generalizability. Also future research is recommended to evaluate PHR usage and its effectiveness with more emphasis on its impact for managing chronic diseases.

\section{Conclusion}

This study has discussed the perceived challenges and barriers for implementation and adoption of the PHR at MNGHA. Even though participants indicated an interest in having a PHR and had no concerns about online accessibility of their health information, there remained an overall concern amongst participants on perceived challenges/barriers such as patient awareness, policy barriers in terms of PHR content, other regulations as a part of the existing business process, and the integration at certain circumstances. Moreover, end user readiness to have a PHR was considered as a potential challenge after its implementation which could hinder its adoption. Therefore it is absolutely crucial in building an efficient PHR addressing end user concerns to ensure a strong adoption rate. Furthermore, effective methods should be utilized in building PHR awareness, setting clear guidelines and regulations for use and involving policy makers prior to implementing the PHR

\section{Acknowledgements}

This study supported by King Abdullah Medical Research Center (KAIMRC) for research grant (SP14/049). 


\section{Conflict of Interest}

The researchers stated that, there are no conflicts of interest.

\section{References}

1. Kupchunas WR. 2007. Personal health record: new opportunity for patient education. $\begin{array}{llll}\text { Orthop } & \text { Nurs. } & \text { 26(3), } & \text { PubMed }\end{array}$ http://dx.doi.org/10.1097/01.NOR.0000276971.86937.c4

2. Bonander J, Gates S. 2010. Public health in an era of personal health records: opportunities for innovation and new partnerships. J Med Internet Res. 12(3), e33. PubMed http://dx.doi.org/10.2196/jmir.1346

3. Archer N, Fevrier-Thomas U, Lokker C, McKibbon KA, Straus SE. 2011. Personal Health Records: A Scoping Review. J Am Med Inform Assoc. 18(4), 515-22. PubMed http://dx.doi.org/10.1136/amiajnl-2011-000105

4. Bates DW, Wells S. 2012. Personal health records and health care utilization. JAMA. 308(19), 2034-36. PubMed http://dx.doi.org/10.1001/jama.2012.68169

5. Detmer D, Bloomrosen M, Raymond B, Tang B. 2008. Integrated Personal Health Records: Transformative Tools for Consumer-centric Care. BMC Med Inform Decis Mak. 8, 45. PubMed http://dx.doi.org/10.1186/1472-6947-8-45

6. Tenforde M, Jain A, Hickner J. 2011. The value of personal health records for chronic disease management: what do we know? Family Medicine-Kansas City. 43(5), 351. PubMed

7. Studeny J, Coustasse A. 2014. Personal health records: is rapid adoption hindering interoperability? Perspect Health Inf Manag. 11(Summer). PubMed

8. Britto MT, Wimberg J. 2009. Pediatric personal health records: current trends and key challenges. Pediatrics. 123(Supplement 2), S97-99. $\quad$ PubMed http://dx.doi.org/10.1542/peds.2008-1755I

9. Tang PC, Ash JS, Bates DW, Overhage JM, Sands DZ. 2006. Personal health records: definitions, benefits, and strategies for overcoming barriers to adoption. $J$ Am Med Inform Assoc. 13(2), 121-26. PubMed http://dx.doi.org/10.1197/jamia.M2025

10. Gaskin GL, Longhurst CA, Slayton R, Das AK. 2011. Sociotechnical challenges of developing an interoperable personal health record: lessons learned. Appl Clin Inform. 2(4), 406. PubMed http://dx.doi.org/10.4338/ACI-2011-06-RA-0035

11. Agarwal R, Anderson C, Zarate J, Ward C. 2013. If We Offer it, Will They Accept? Factors Affecting Patient Use Intentions of Personal Health Records and Secure Messaging. J Med Internet Res. 15(2), e43. PubMed http://dx.doi.org/10.2196/jmir.2243 
12. Bitton A, Onega T, Tosteson AN, Haas JS. 2014. Consumer Perspectives on Personal Health Records: A 4-Community Study. Am J Manag Care. 20(4), 287-96. PubMed

13. Kahn JS, Aulakh V, Bosworth A. 2009. What it takes: characteristics of the ideal personal health record. Health Aff. 28(2), 369-76. PubMed http://dx.doi.org/10.1377/hlthaff.28.2.369

14. Pottas D, Mostert-Phipps N. 2012. Citizens and personal health records-the case of Nelson Mandela bay. Stud Health Technol Inform. 192, 501-04. PubMed

[15] Halamka, J. D., Mandl, K. D., \& Tang, P. C. (2008). Early experiences with personal health records. Journal of the American Medical Informatics Association, 15(1), 1-7.

[16] Lober, W.B., Zierler, B., Herbaugh, A., Shinstrom S.E., Stolyar., Kim, E.H.,\& Kim, Y. (2006) Barriers to the use of a Personal Health Record by an Elderly Population. Journal of the Schools of Nursing \&Medicine, 514-518

17. Luque AE, van Keken A, Winters P, Keefer MC, Sanders M, et al. 2013. Barriers and facilitators of online patient portals to personal health records among persons living with HIV: formative research. JMIR Res Protoc. 2(1). http://dx.doi.org/10.2196/resprot.2302

18. Cocosila M, Archer N. 2014. Perceptions of chronically ill and healthy consumers about electronic personal health records: a comparative empirical investigation. BMJ Open. 184(7), e005304. PubMed http://dx.doi.org/10.1136/bmjopen-2014-005304

19. King Abdullah International Medical Research Center. Available from:http://www.ksauhs.edu.sa/English/KnowledgeCenter/Pages/KingAbdullahMedicalResearchCenter.aspx [Accessed: December 1, 2014]

20. Topline Results from a National Consumer Survey on Health IT (2010). Retrieved December 8, 2013 from Lake Research Parteners, Web site: http:/www.chcf.org/ /media/MEDIA\%20LIBRARY\%20Files/PDF/T/PDF\%20ToplineRes ultsNationalConsumerSurveyHIT.pdf

21. AlKabba A, Hussein G, Albar A, Bahnassy A, Qadi M. 2012. The major medical and ethical challenges facing the public and healthcare providers in Saudi Arabia. J Family Community Med. 19(1), 1-6. PubMed http://dx.doi.org/10.4103/2230-8229.94003

22. AlMutair A. 2014. Attitudes of healthcare providers towards family involvement and presence towards family involvement and presence in adult critical care units in Saudi Arabia: a quantitative study. $J$ Clin Nurs. 23(5-6), 744-55. http://dx.doi.org/10.1111/jocn.12520

23. Emani S, Yamin C, Peters E, Karson A, Lipsitz S, et al. 2012. Patient perceptions of a personal health record: A test of the Diffusion of Innovation Model. J Med Internet Res. 14(6), e150. PubMed http://dx.doi.org/10.2196/jmir.2278 
[24] Research Data Management - King Abdullah International Medical Research Center (MNGHA). Data retrieved 26 ${ }^{\text {th }}$ October 2016. 